\title{
Design and Simulation of Photonic Crystal Fiber Based Surface Plasmon Resonance Biosensor for Cancer Diagnosis
}

\section{Mehdi Fazeli}

Islamic Azad University of Arak

Ashkan Horri ( $\square$ ashkan.horri@srbiau.ac.ir )

Deparment of Electrical Engineering, Arak Branch, Islamic azad University, Arak Iran https://orcid.org/0000-0003-4187-8883

\section{Research Article}

Keywords: Biosensor, photonic crystal fiber, Surface plasmon, Finite element method, Plasmonic Material, surface plasmon resonance sensor

Posted Date: April 16th, 2021

DOI: https://doi.org/10.21203/rs.3.rs-397327/v1

License: (c) (i) This work is licensed under a Creative Commons Attribution 4.0 International License. Read Full License 


\title{
Design and Simulation of Photonic Crystal Fiber Based Surface Plasmon Resonance Biosensor for Cancer Diagnosis
}

\section{Authors:}

\section{1-Mehdi fazeli}

Affiliation: Department of Electrical Engineering, Arak Branch, Islamic Azad university, Arak, Iran.

Email: mehdi.fazeli.mut@gmail.com

\section{2-Ashkan Horri}

Affiliation: Department of Electrical Engineering, Arak Branch, Islamic Azad university, Arak, Iran.

Email: ashkan.horri@srbiau.ac.ir \& ashkan_horri@yahoo.com

\begin{abstract}
A surface plasmon resonance (SPR) sensor based on photonic crystal fiber (PCF) was designed and gold metal was used as a sensitive plasmonic layer. Performance of this sensor was investigated using the finite element method (FEM) for refractive indexes (RI) of 1.33 to 1.41 . This sensor has a sensitivity for the defined refractive indexes range between 2600 to $28921 \mathrm{~nm} / \mathrm{RIU}$ at wavelengths of 650 to $1400 \mathrm{~nm}$ and has the highest resolution and best FOM with a value of $3.46 \times 10^{-6}$ RIU, $222.45 \mathrm{RIU}^{-1}$, respectively. Diagnosis of cancer by examining blood samples is one of the advantages of this sensor. Due to the simple structure of this sensor and its good performance, it can be used as a good diagnostic tool for analytes with different refractive index.
\end{abstract}

\section{Keywords:}

Biosensor, photonic crystal fiber, Surface plasmon, Finite element method, Plasmonic Material, surface plasmon resonance sensor 


\section{1- Introduction}

The oscillations of the electrons at the interface between the dielectric and the metal are called surface plasmon. These oscillations are caused by the collision of light with electrons. If the frequency of the emitted light is the same as the natural frequency of the electron, the surface plasmon wave propagates along with the dielectric-metal interface, which is called the surface plasmon resonance (SPR) [1]. SPR technology provides access to ultra-compact detection sensors and electronic devices [2]. Due to the sensitivity of SPR sensor to refractive index and label-free sensing capability, this sensor as a direct measuring device has many applications, such as environmental monitoring [3], food health [ 4], biological material detection [5], and film thickness monitoring [6].

Conventional optical sensors made using the SPR phenomenon had problems that were mostly solved with the advent of photonic crystal fiber (PCF) and the use of their advantages such as smaller size, easier light transmission and single-mode light emission. Sensitivity in PCF-based SPR sensors can be adjusted in PCF by using the number of rings and air-hole dimensions [7]. The SPR phenomenon is created using gold, silver, copper and aluminum in most PCF-based sensors today. Among them, gold is more compatible with organic environments [8]. PCF-based SPR sensors are generally divided into two categories. In the first category, metal layer coating is selectively placed inside the air holes created in the PCF and the analyte material is optionally placed in the same holes. In the second category, metal coating is placed in the outer layer of PCF and the analyte material is placed on it. The first category is less favorite since the placement of the metal layer inside the air holes is difficult, but the second category is used more because the placement of the metal layer on the outer surface is easier and has a good sensitivity [9-11]. However, so far sensors have been designed that have used the first type of fabrication method [12,13]. In 2019, for

example, Chao Liu et al. proposed an SPR sensor that used a gold nanowire as a plasmonic sensitive layer and achieved a sensitivity of 14200 [14]. In another paper, Zhenkai Fan (2019) presented a structure, which used a gold ring inside the PCF as a 
plasmonic layer and was able to achieve a sensitivity of 2150 [15]. Also, Suoda Chu et al. (2020) proposed a PCF-based SPR sensor that used two silver nanowires in air holes. At best, the sensor offered a sensitivity of 3400 and a resolution of 2.5 [16].

One of the applications that has always been suggested for SPR sensors is its use as a diagnostic tool. Among diseases, leukemia is one of the most common diseases in the world today, which has different types and kills thousands of people every year. However, accurate and quick diagnosis can help a lot in its treatment. Biosensors made using PCF can be used in research on cancer cells [17-18].

This paper presents a PCF-based SPR sensor. The performance of this proposed sensor was evaluated using LUMERICAL software in the refractive index range of 1.33 to 1.41 . In addition, the function of this sensor for detection of several types of cancer was evaluated.

\section{2- Sensor design and numerical simulation}

A schematic of the proposed PCF-based SPR biosensor is shown in Figure 1. The PCF sensor can be fabricated by the die-cast and stack-and-draw methods [19,20] As shown in the figure, this biosensor consists of a regular hexagon composed of air holes on the bed of Fused Silica with a diameter of $16 \mu \mathrm{m}$, and the diameter of the air holes $\mathrm{d} 2, \mathrm{~d} 3, \mathrm{~d} 4$ and $\mathrm{d} 5 \mathrm{is} .8 \mu \mathrm{m}, 1.2 \mu \mathrm{m}, 1.6 \mu \mathrm{m}$, and $2 \mu \mathrm{m}$, respectively. The distance between the air holes is called the lattice pitch and is denoted by $\mathbf{A}$, which is equal to $3 \mu \mathrm{m}$. A layer of gold with a thickness of $40 \mathrm{~nm}$ and a length of $7 \mu \mathrm{m}$ is used as a surface plasmon excitation layer. The distance from the gold layer to the center of the core is $4.4 \mu \mathrm{m}$. The thickness of the analyte layer in part $\mathrm{d} 1$ is equal to $1.6 \mu \mathrm{m}$. 


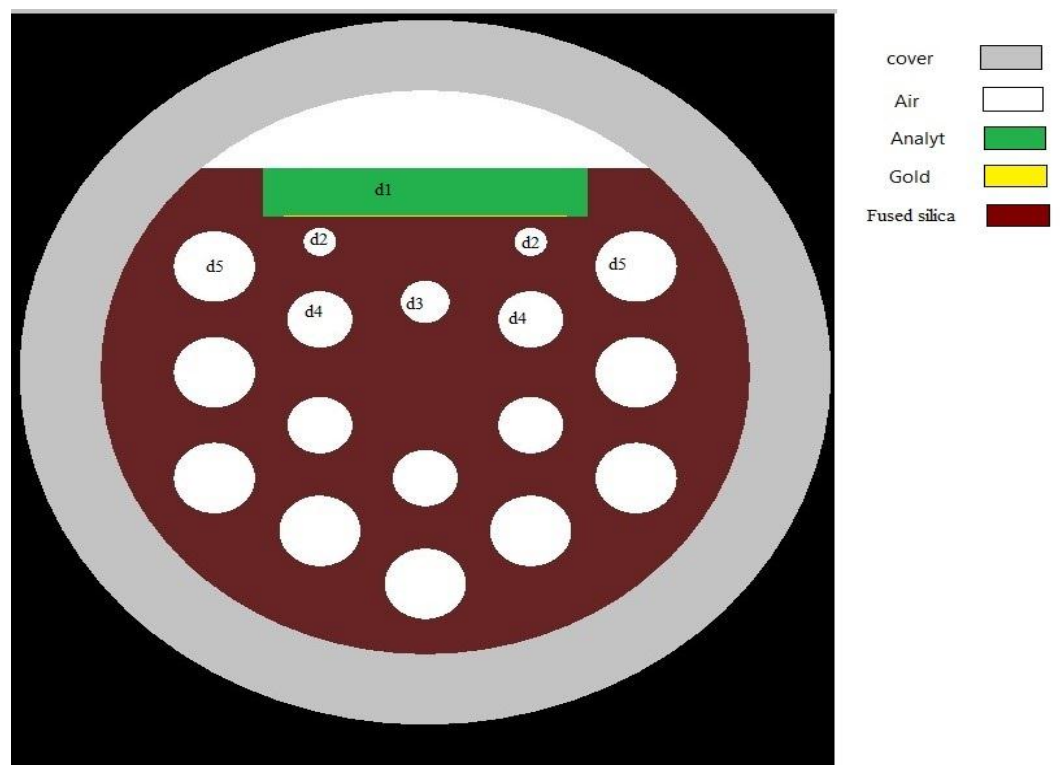

Fig. 1. Cross-section of the proposed PCF-based SPR biosensors

The function of this sensor is that the analyte solution is in contact with the smooth and polished surface of gold, and when light is concentrated in the center of the core, some losses occur in the gold layer. Because the analyte refractive index is varied, the peak of these losses occurs each time at a specific wavelength. In this way, a spectrum can be obtained by optical spectrum analysis (OSA).

Fused Silica is used as the core coating material and its refractive index is calculated from the Sellmeier equation [21],

$\mathrm{n}(\lambda)^{2}=1+\frac{B_{1}^{2} \lambda^{2}}{\lambda^{2}+C_{1}}+\frac{B_{2}^{2} \lambda^{2}}{\lambda^{2}+C_{2}}+\frac{B_{3}^{2} \lambda^{2}}{\lambda^{2}+C_{3}}$

Where the values of $\mathrm{B}_{1}, \mathrm{~B}_{2}, \mathrm{~B}_{3}, \mathrm{C}_{1}, \mathrm{C}_{2}, \mathrm{C}_{3}$, are 0.691663, 0.407943, 0.897479, $0.004679 \mu \mathrm{m}^{2}, \quad 0.013512 \mu \mathrm{m}^{2}$, and $97.934003 \mu \mathrm{m}^{2}$, respectively; and $\lambda$ is the wavelength of light emitted in the center of the fiber core. Also, the dielectric constant of the gold layer is calculated using the Drude model [21].

$\varepsilon=\varepsilon_{\infty}-\frac{w_{p}^{2}}{w\left(w+j w_{c}\right)}$

here $\varepsilon_{\infty}=9.75$ is the high frequency dielectric function of gold; while $\mathrm{w}_{\mathrm{p}}=1.36 \times 10^{16}$ $\mathrm{rad} / \mathrm{s}$ and $\mathrm{w}_{\mathrm{c}}=1.45 \times 10^{14} \mathrm{rad} / \mathrm{s}$ are the plasma frequency of the metal and scattering 
frequency of the electrons, respectively. The air refractive index is considered one [21].

To evaluate the performance of this biosensor, the finite element method (FEM) is used by LUMERICAL software.

\section{3- Results and discussion}

The principle of operation of PCR-based SPR sensor is based on the interaction between electrons on the surface between the metal and the insulation and the field created around it. When a light wave propagates through the PCF core, a field is created which, when it collides with an electron on the surface of the metal layer, creates a surface plasmon wave (SPW). If the fundamental mode (FM) frequency of the light guided in the nucleus coincides with the surface plasmon polariton (SPP) mode frequency, phase matching occurs and a peak of losses occurs at that frequency $[22,23]$. In this sensor, the perimeter of the metal layer is filled with analyte, and when there are small changes in the refractive index of the analyte around the metal layer, the position of the effective refractive index changes according to the wavelength, resulting in peak losses in the phase shift mode.

The simulation results for the proposed PCF-based SPR sensor are shown in Figure 2. As you can see, Figure 2 shows the field distribution well in FM and SPP modes and phase matching in the range of $650-1400 \mathrm{~nm}$ for analytes with a refractive index of 1.4. In this figure, $2 a, 2 b$, and $2 c$ are the field distributions in FM, SPP, and the phase matching modes, respectively. 


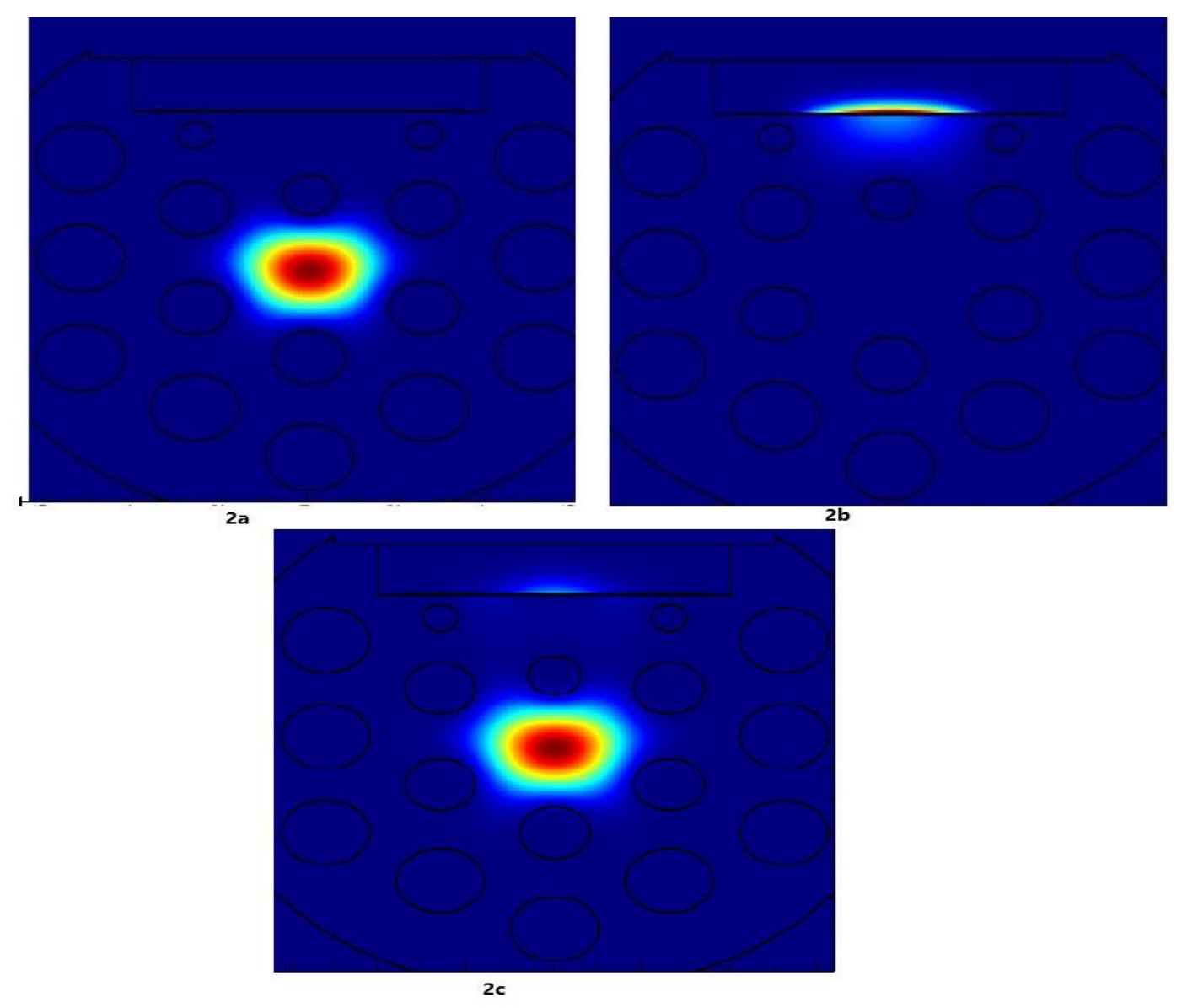

Fig.2. Light energy flow distributions for the analyte with $n=1.4,2 a$ ) are the field distributions in FM mode, 2b) SPP mode, 2c) phase matching modes

Figure 3 shows the spectrum of losses generated (blue curve), scattering relationship, FM mode (green curve), and surface plasmon polariton (SPP) mode (red curve). The amount of losses in the plasmonic layer is calculated in $\mathrm{dB} / \mathrm{cm}$ by the following formula [21]:

$\alpha_{\text {loss }}=8.686 \times \mathrm{K}_{0} \times \operatorname{Im}\left[\mathrm{n}_{\mathrm{eff}}\right] \times 10^{4}$

Where $\operatorname{Im}\left[\mathrm{n}_{\mathrm{eff}}\right]$ denotes the imaginary part of the refractive index and $\mathrm{K}_{0}=\frac{2 \pi}{\lambda}$ is wave number in which the value of $\lambda$ is in micrometers. As shown in the figure 3 , the phase matching process is performed at a wavelength of $1.0901 \mu \mathrm{m}$ and the highest losses as $14.605 \mathrm{~dB} / \mathrm{cm}$ occur at this wavelength for analytes with a refractive index of 1.4, showing that the highest energy transfer from FM mode to SPP mode occurs at this wavelength and causes resonance and production of surface plasmon. 


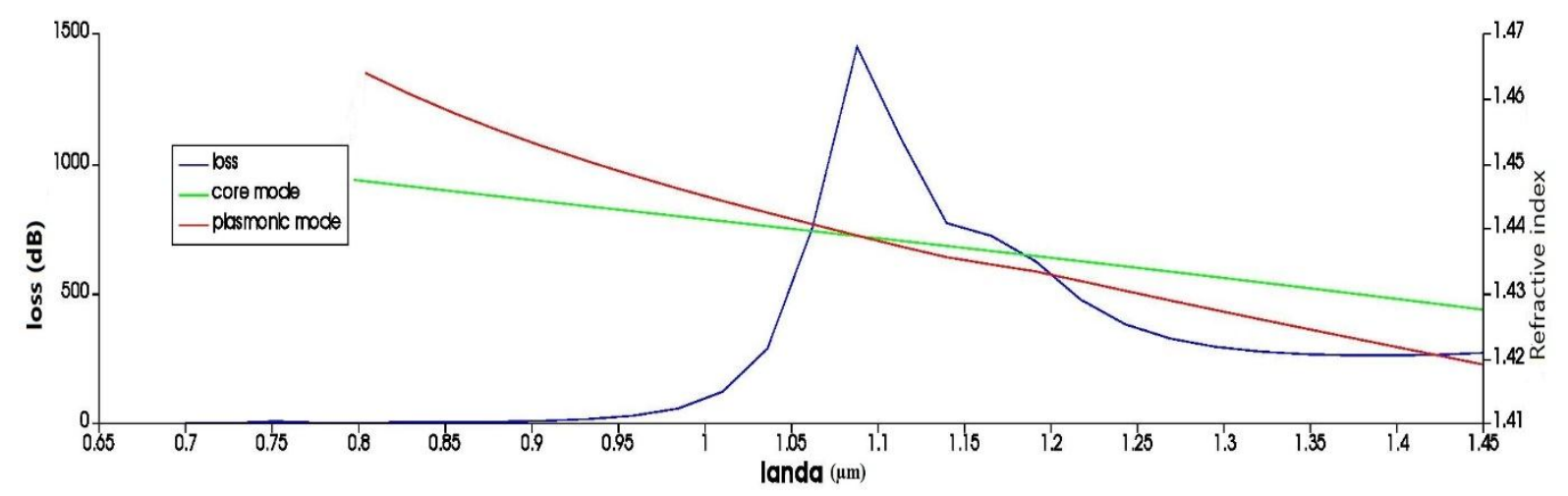

Fig.3. The confinement loss, dispersion relations of FM and plasmonic mode for $n_{a}=1.4$

The performance of this sensor for different analyte refractive index (1.33 to 1.41) is shown in Figure 4. As shown in the figure, the maximum wavelength shift occurs between the refractive index 1.4 to 1.41

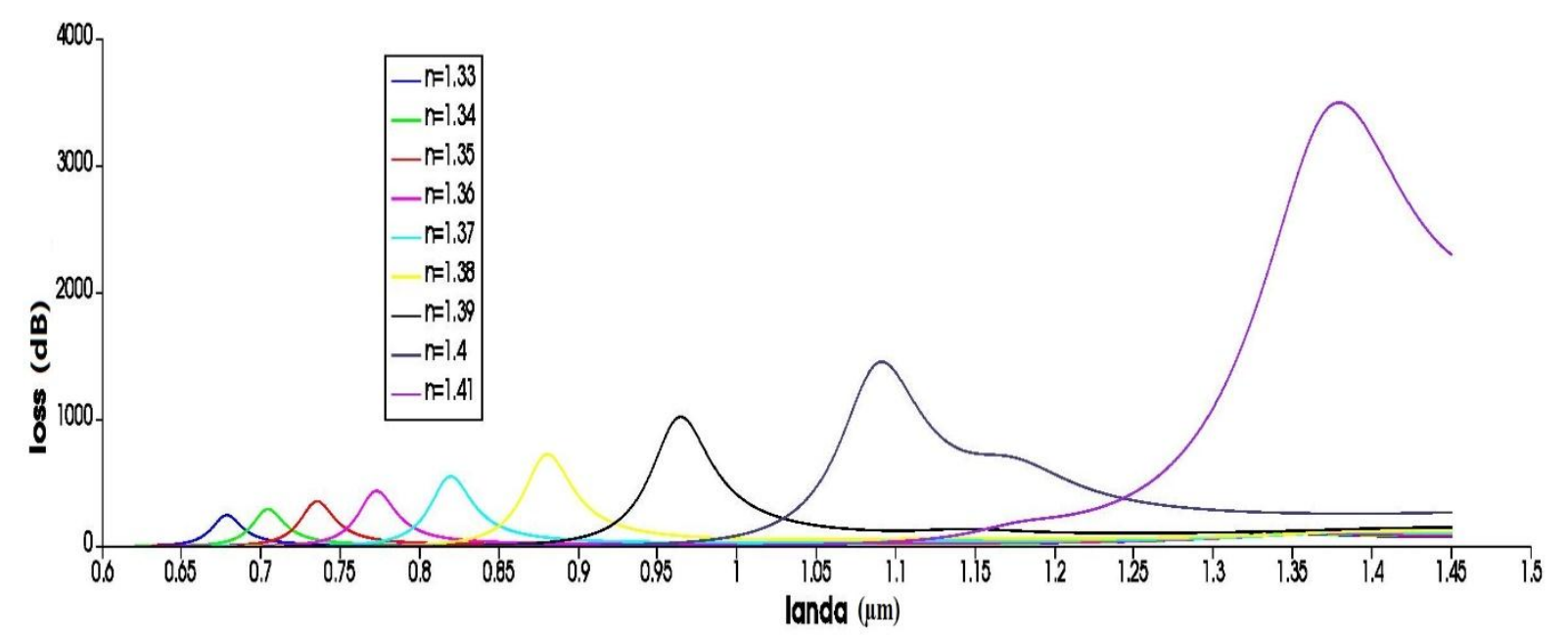

Fig.4. Loss spectra with increasing analyte RI from 1.33 to 1.41

The performance of this sensor is mainly determined by structural parameters, such as resonance wavelength, losses, and sensitivity [24,19]. The sensitivity of the biosensor can be obtained by the interrogation method in terms of nm/RIU by the following formula [21]:

$\mathrm{S}_{\lambda}(\mathrm{nm} / \mathrm{RIU})=\frac{\Delta \lambda p e a k}{\Delta n a}$ 
Where, $\Delta \lambda$ is the amount of resonance wavelength displacement and $\Delta \mathrm{n}$ is the difference of the analyte refractive index. The maximum resonance wavelength shift is $289.21 \mathrm{~nm}$ and occurs to change the refractive index from 1.4 to 1.41 with a sensitivity value of 28921. Sensor resolution is another important parameter obtained by the following formula [19]:

$\mathrm{R}(\mathrm{RIU})=\Delta \mathrm{n}_{\mathrm{a}} \frac{\Delta \lambda m i n}{\Delta \lambda p e a k}$

Where, $\Delta \lambda_{\text {peak }}$ is the maximum resonant wavelength peak shift, $\Delta \lambda_{\min }=0.1(\mathrm{~nm})$ is the minimum spectral resolution, $\Delta \mathrm{n}=0.01$ is the refractive index changes. For example, to change the refractive index from 1.4 to 1.41 , the value of $\Delta \lambda_{\text {peak }}=289.21 \mathrm{~nm}$, whose resolution value is $3.46 \times 10^{-6} \mathrm{RIU}$. The relationship between the resolution and the refractive index of the analyte is shown in Figure 5.

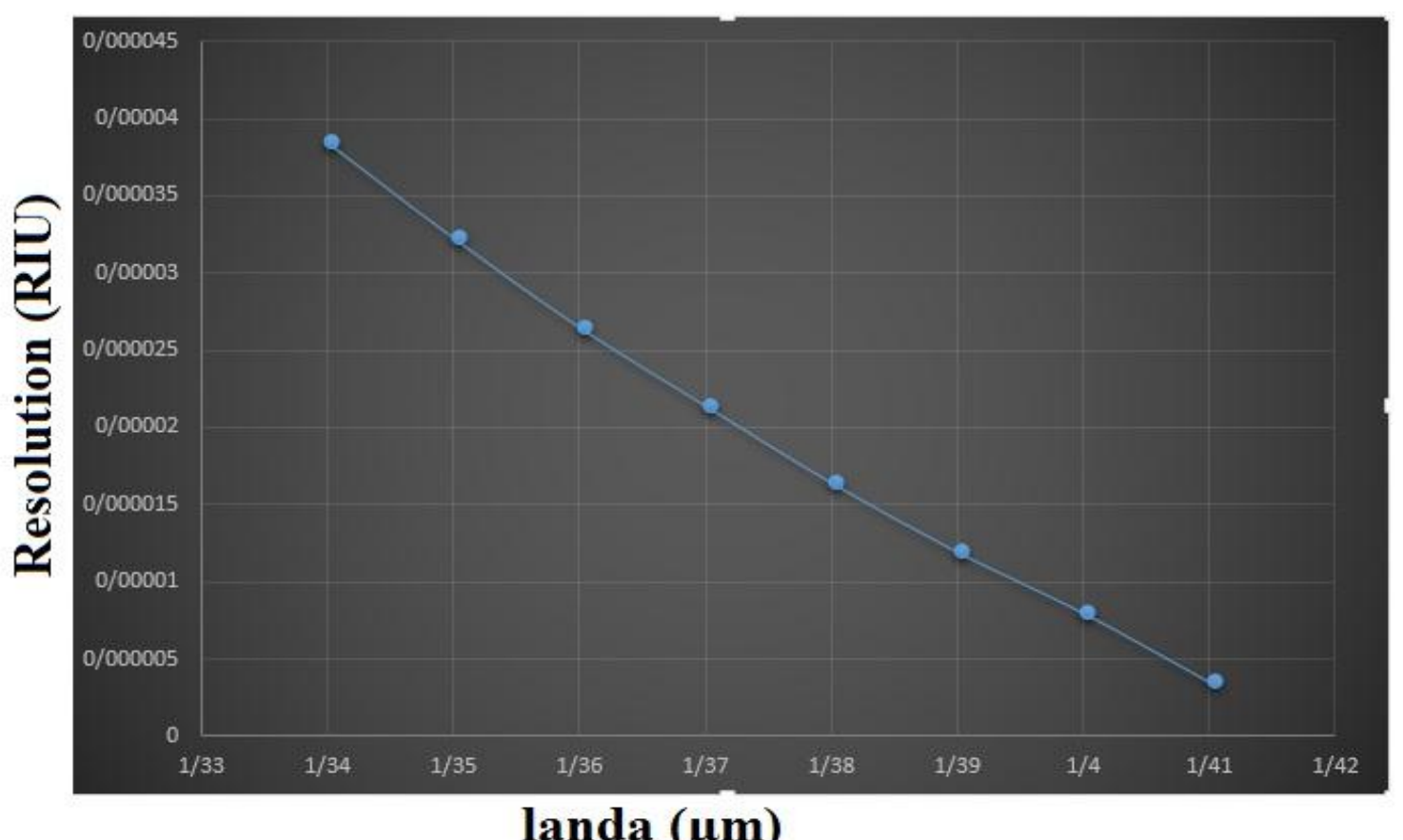

Fig. 5. Resolution versus for different analyte's RI

The figure of merit (FOM) is another parameter that is calculated for the sensor by the following formula [21]:

$\mathrm{FOM}\left(\mathrm{RIU}^{-1}\right)=\frac{s\left(\frac{n m}{R I U}\right)}{F W H M(n m)}$ 
Where, $\mathrm{S}$ is sensor sensitivity and FWHM denotes full bandwidth at half-maximum. a higher value of FOM responsible to a better detection limit. The relationship between FOM and refractive index is shown in Figure 6.

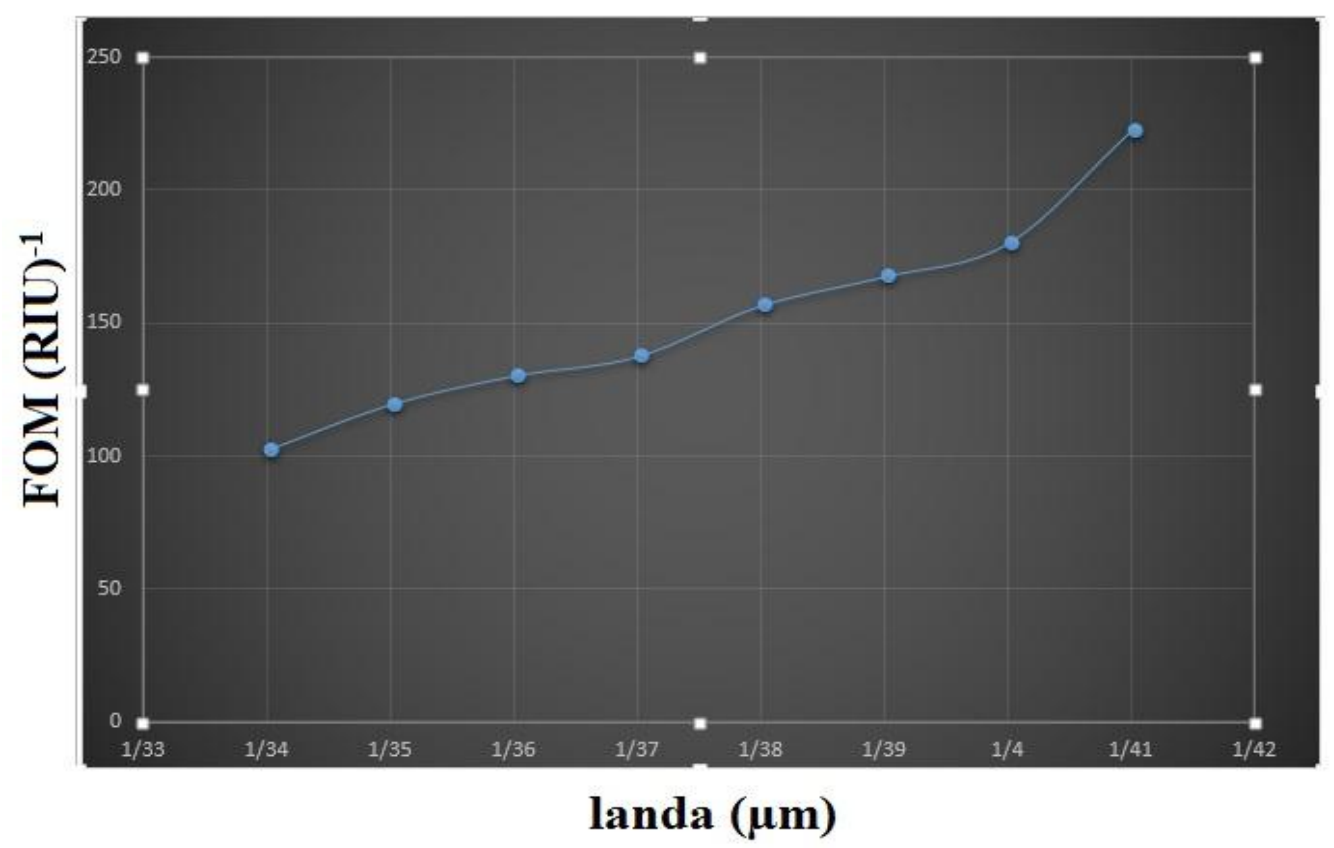

Fig.6. FOM versus for different analyte's RI

Table 1 shows the resonance wavelengths, sensitivity, resolution, and FOM for this sensor at different refractive indexes.

Table.1. Properties of the proposed biosensor

\begin{tabular}{lcclcccc}
$\begin{array}{l}\text { RI of } \\
\text { Analyte }\end{array}$ & $\begin{array}{l}\text { peak loss } \\
(\mathbf{d B} / \mathbf{c m})\end{array}$ & $\begin{array}{l}\text { resonance wave } \\
\text { length }(\mathbf{n m})\end{array}$ & $\begin{array}{l}\text { FWHM } \\
(\mathbf{n m})\end{array}$ & $\begin{array}{c}\text { peak } \\
\text { shift }(\mathbf{n m})\end{array}$ & $\begin{array}{c}\text { sensivity } \\
(\mathbf{n m} / \mathbf{R I U})\end{array}$ & $\begin{array}{c}\text { Resolution } \\
(\mathbf{R I U})\end{array}$ & $\begin{array}{c}\text { FOM } \\
(\mathbf{R I U})^{-1}\end{array}$ \\
\hline 1.33 & 2.52 & 678 & 24 & Ref & Ref & - & - \\
1.34 & 2.98 & 704 & 25.5 & 26 & 2600 & $3.85 \times 10^{-5}$ & 101.96 \\
1.35 & 3.61 & 735 & 26 & 31 & 3100 & $3.23 \times 10^{-5}$ & 119.23 \\
1.36 & 4.43 & 772.7 & 29 & 37.7 & 3770 & $2.65 \times 10^{-5}$ & 130 \\
1.37 & 5.579 & 819.38 & 34 & 46.68 & 4668 & $2.14 \times 10^{-5}$ & 137.29 \\
1.38 & 7.328 & 880.4 & 39 & 61.02 & 6102 & $1.64 \times 10^{-5}$ & 156.46 \\
1.39 & 10.282 & 964.1 & 49 & 83.7 & 8370 & $1.19 \times 10^{-5}$ & 170.82 \\
1.4 & 14.605 & 1090.1 & 70 & 125.9 & 12590 & $7.94 \times 10^{-6}$ & 179.86 \\
1.41 & 35.04 & 1379.31 & 130 & 289.21 & 28921 & $3.46 \times 10^{-6}$ & 222.45
\end{tabular}


Basal cancer cells are caused by exposure to strong sunlight on the outer layer of the skin. The refractive index of this cancer cell is 1.38 and the refractive index of healthy base cells is 1.36 . HeLa cancer cells are another human cancer cell. This cancer is caused by the infection of cells with the human papilloma virus 18 (HPV18). The refractive index of Hela cancer cells is 1.392 , while the refractive index of normal HeLa cells is 1.368. MDA-MB-231 is another type of cancer cell found in a patient with breast cancer. Refractive index of its normal type is 1.385 and its cancer cell refractive index is 1.399 [18].

Figure 7 shows the function of the sensor proposed for the cancer cells described above.

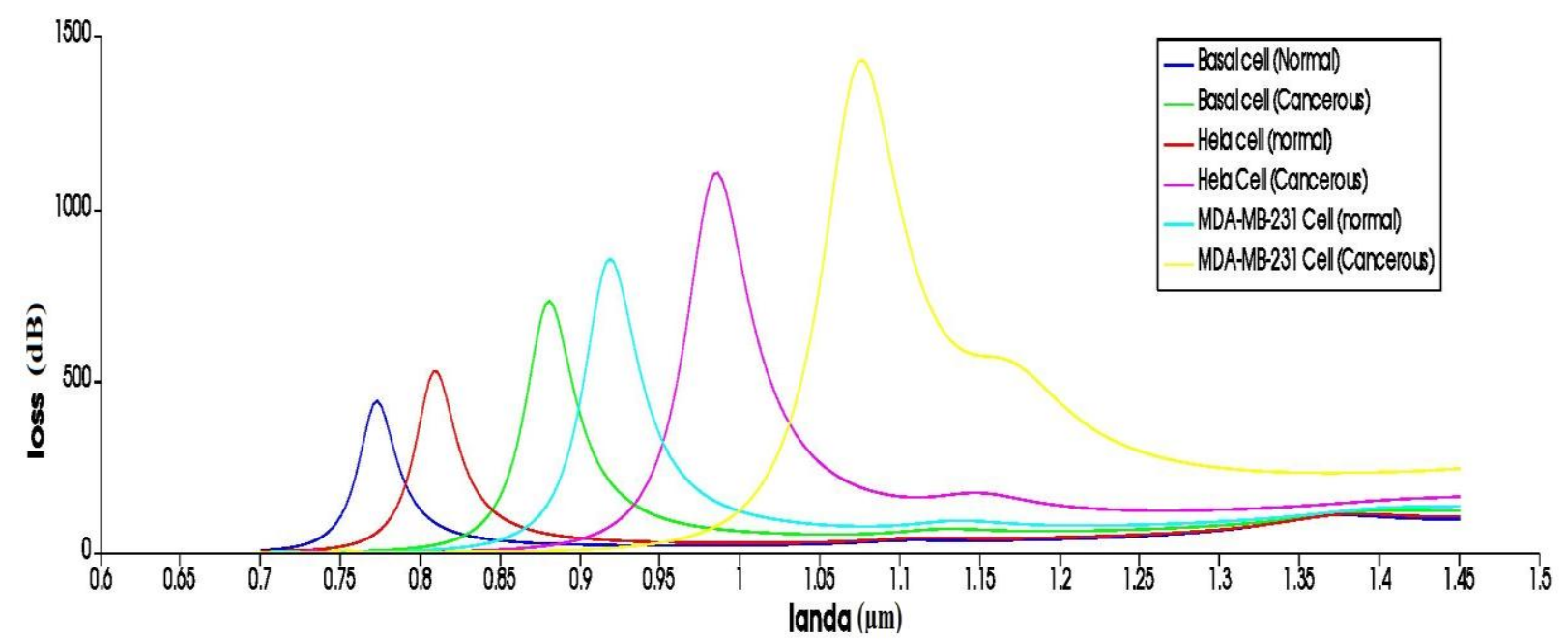

Fig.7. Loss spectra for Normal and cancer cell

A comparison of the sensor output for each of the normal and cancer cells is given in Table 2. As can be seen, this sensor has a good function for detecting this type of cancer. 
Table.2. performance biosensor for diagnosis cancer cell

\begin{tabular}{|c|c|c|c|c|c|c|c|c|}
\hline $\begin{array}{l}\text { Analyte } \\
\text { use }\end{array}$ & RI & $\begin{array}{l}\text { peak loss } \\
(\mathrm{dB} / \mathrm{cm})\end{array}$ & $\begin{array}{c}\text { resonance wave } \\
\text { length }(\mathbf{n m})\end{array}$ & $\begin{array}{c}\text { FWHM } \\
(\mathbf{n m})\end{array}$ & $\begin{array}{l}\text { peak } \\
\text { shift }(\mathbf{n m})\end{array}$ & $\begin{array}{l}\text { sensivity } \\
\text { (nm/RIU) }\end{array}$ & $\begin{array}{c}\text { Resolution } \\
\text { (RIU) }\end{array}$ & $\begin{array}{r}\text { FOM } \\
(\text { RIU })^{-1}\end{array}$ \\
\hline Basal cell (Normal) & 1.36 & 4.433 & 772.7 & 29 & Ref & Ref & - & - \\
\hline Basal cell (Cancerous) & 1.38 & 7.328 & 880.4 & 39 & 107.7 & 5385 & $1.86 \times 10^{-5}$ & 138.08 \\
\hline Hela cell (normal) & 1.368 & 5.31 & 809.01 & 33 & Ref & Ref & - & - \\
\hline Hela Cell (Cancerous) & 1.392 & 11.05 & 984.97 & 52 & 175.96 & 7331.7 & $1.36 \times 10^{-5}$ & 141 \\
\hline $\begin{array}{l}\text { MDA-MB-231 } \\
-\end{array}$ & 1.385 & 8.55 & 918.48 & 42 & Ref & Ref & - & \\
\hline Cell (normal) & & & & & & & & \\
\hline $\begin{array}{l}\text { MDA-MB-231 } \\
\text { Cell (Cancerous) }\end{array}$ & 1.399 & 14.327 & 1075.79 & 69 & 157.31 & 11236.4 & $8.9 \times 10^{-6}$ & 162.85 \\
\hline
\end{tabular}

To evaluate the overall performance of this sensor, Table 3 compares the properties of the proposed PCF-based SPR biosensor with the structures found in other articles. In this comparison, the sensitivity, refractive index measurement range, resolution, and FOM of the sensor are considered. As can be seen from the table, the proposed SPR PCF sensor performs better in terms of sensitivity, resolution and FOM.

Table.3. Comparison among the properties of the proposed biosensor with previously reported sensors.

\begin{tabular}{lcccc}
\hline Ref & Detection RI range & sensivity $(\mathbf{n m} / \mathbf{R I U})$ & Resolution(RIU) & FOM $(\mathbf{R I U})^{-1}$ \\
\hline$[14]$ & $1.33-1.4$ & 14200 & $7.04 \times 10^{-6}$ & - \\
{$[15]$} & $1.33-1.43$ & 2150 & - & - \\
{$[16]$} & $1.35-1.50$ & 3400 & $2.94 \times 10^{-5}$ & - \\
{$[19]$} & $1.22-1.33$ & 15000 & $6.67 \times 10^{-6}$ & - \\
{$[21]$} & $1.40-1.44$ & 17000 & - & 62.2 \\
{$[25]$} & $1.33-1.41$ & 17000 & $5.88 \times 10^{-6}$ & - \\
{$[26]$} & $1.33-1.42$ & 21000 & $4.76 \times 10^{-6}$ & - \\
{$[27]$} & $1.42-1.46$ & 15000 & - & 45 \\
{$[28]$} & $1.33-1.37$ & 10000 & $1 \times 10^{-5}$ & - \\
{$[29]$} & $1.27-1.37$ & 22400 & - & 127 \\
Proposed work & $1.33-1.41$ & 28921 & $3.46 \times 10^{-6}$ & 222.45 \\
\hline
\end{tabular}




\section{4- Conclusion}

In this paper, we propose a D-shaped PCF-based SPR sensor for measuring analytes with a refractive index of 1.33 to 1.41 . In this sensor, gold metal was used as a sensitive plasmonic layer. The simulation results show that the maximum sensitivity for this sensor in the tested range is $28921 \mathrm{~nm} / \mathrm{RIU}$. Also, its maximum resolution and best FOM is $3.46 \times 10^{-6} \mathrm{RIU}$ and $222.45 \mathrm{RIU}^{-1}$, respectively. The proposed sensor has the ability to distinguish a person with cancer from a healthy person by testing a blood sample.

\section{5- Reference}

[1]P. K. Maharana, P. Padhy and R. Jha; "On the field enhancement and performance of an ultrastable SPR biosensor based on grapheme," IEEE Photonics Technoogy. Lett., vol. 25, no. 22, pp. 2156-2159, 2013.

[2] S.P. Burgos, H.W. Lee, E. Feigenbaum, et al., Synthesis and characterization of plasmonic resonant guided wave networks, Nano Lett. 14 (6) (2014)3284e3292.

[3] Kim YC, Cramer J, Battaglia T, Jordan JA, Soame NB, Peng W,Laurel LK, Karl SB (2013) Investigation of in situ surface plasmon resonance spectroscopy for environmental monitoring in and around deep-sea hydrothermal vents. Anal Lett 46(10):1607-1617

[4] Chen ST, Mark HM, Christopher TE, Jos B (2010) Advances in surface plasmon resonance biosensor technology towards highthroughput, food-safety analysis. TrAC Trends Anal Chem 29(11):1305-1315

[5] Li L, Zhang X, Liang Y, Guang J, Peng W (2016) Dual-channel fiber surface plasmon resonance biological sensor based on a hybrid interrogation of intensity and wavelength modulation. J Biomed Opt. doi:10.1117/1.JBO.21.12.127001

[6] T. Akimoto, S. Sasaki, K. Ikebukuro, et al., Refractive-index and thickness sensitivity in surface plasmon resonance spectroscopy, Appl. Optic. 38 (19) (1999) 4058e4064.

[7] R. K. Gangwar and V. K. Singh, "Highly sensitive surface plasmon resonance based Dshaped photonic crystal fiber refractive index sensor," Plasmonics, vol. 12, no. 5, pp. 13671372, 2017.

[8] A. A. Rifat, G. A. Mahdiraji, Y. M. Sua, Y. G. Shee, R. Ahmed, D. M. Chow and F. M. Adikan, "Surface plasmon resonance photonic crystal fiber biosensor: a practical sensing approach," IEEE Photonics Technology Letters, vol. 27, no. 15, pp. 1628- 1631, Aug. 2015. 
[9] M. R. Hasan, S. Akter, K. Ahmed and D. Abbott, "Plasmonic refractive index sensor employing niobium nanofilm on photonic crystal fiber," IEEE Photonics Technology Letters, vol. 30, no. 4, pp. 315-318, Feb. 2018.

[10] X. Yang et al., "Analysis of graphene-based photonic crystal fiber sensor using birefringence and surface plasmon resonance," Plasmonics 12(2), 489-496 (2017)

[11] A. A. Rifat, M. R. Hasan, R. Ahmed and H. Butt, "Photonic crystal fiber-based plasmonic biosensor with external sensing approach," Journal of Nanophotonics, vol. 12, no. 1, pp. 012503, Jun. 2017.

[12] A. Rifat et al., "Photonic crystal fiber-based surface plasmon resonance sensor with selective analyte channels and graphene-silver deposited core," Sensors 15(5), 11499-11510 (2015).

[13] W. Qin et al., "Analyte-filled core self-calibration microstructured optical fiber based plasmonic sensor for detecting high refractive index aqueous analyte," Opt. Lasers Eng. 58, $1-8(2014)$.

[14] Chao Liu, Guanglai Fu, Famei Wang, Zao Yi, Chunhong Xu, Lin Yang, Qiang Liu, Wei Liu, Xianli Li, Haiwei Mu, Tao Sun, Paul K. Chu," Ex-centric core photonic crystal fiber sensor with gold nanowires based on surface plasmon resonance" Optik, Volume 196, November 2019, 163173

[15] Zhenkai Fan, "Surface plasmon resonance refractive index sensor based on photonic crystal fiber covering nano-ring gold film" Optical Fiber Technology, Volume 50, July 2019, Pages 194-199

[16] Suoda Chu ; K. Nakkeeran ; Abdosllam M. Abobaker ; Sumeet S. Aphale ; S. Sivabalan ; P. Ramesh Babu and K. Senthilnathan. " A Surface Plasmon Resonance Bio-Sensor based on Dual Core D-Shaped Photonic Crystal Fibre Embedded with Silver Nanowires for MultiSensing" : IEEE Sensors Journal ,Volume: 21, Issue: 1, Jan.1, 12021

[17] Erim, Nur, Muhammed Necip Erim, and Hamza Kurt. "An Optical Sensor Design Using Surface Modes of Low Symmetric Photonic Crystals." IEEE Sensors Journal. Volume: 19, Issue: 14, 26 March 2019.

[18] Mojtaba Hosseinzadeh Sani, Saeed Khosroabadi. "A novel design and analysis of high sensitivity biosensor based on nano-cavity for detection of blood component, diabetes, cancer and glucose concentration" IEEE Sensors Journal, Volume: 20,Page(s): 7161 - 7168 , 06 January 2020

[19] Chao Liu, Jianwei Wang, Famei Wang, Weiquan Su, Lin Yang, Jingwei Lv ,Guanglai Fu, Xianli Li, Qiang Liu, Tao Sun, Paul K. Chu . " Surface plasmon resonance (SPR) infrared sensor based on D-shape photonic crystal fibers with ITO coatings" Optics Communications, Volume 464, 1 June 2020, 125496

[20] G.Y. Zhou, Z.Y. Hou, S.G. Li, L.T. Hou," Fabrication of glass photonic crystal fibers with a die-cast process", Appl. Opt. 45 (2006) 4433-4436. 
[21] Suoda Chu, K. Nakkeeran, Senior, Abdosllam M. Abobaker,, Sumeet S. Aphale,Senior, P. Ramesh Babu and K. Senthilnathan" Design and Analysis of Surface-Plasmon-ResonanceBased Photonic Quasi-Crystal Fiber Biosensor for High-Refractive-Index Liquid Analytes " , IEEE Journal of Selected Topics in Quantum Electronics, Volume: 25, Issue: 2, March-April 2019

[22] R. Otupiri, E. Akowuah, S. Haxha, et al.," A novel birefrigent photonic crystal fiber surface plasmon resonance biosensor", IEEE Photon. J. 6 (4) (2014) 1e11.

[23] Moriom Rojy Momota, Md. Rabiul Hasan. "Hollow-core silver coated photonic crystal fiber plasmonic sensor" , Optical Materials, Accepted 25 December 2017.

[24] E.X. Liu, W. Tan, B. Yan, J.L. Xie, R. Ge, J.J. Liu, "Broadband ultra-flattened dispersion, ultra-low confinement loss and large effective mode area in an octagonal photonic quasi-crystal fiber", J. Opt. Soc. Am. A 35 (2018) 431-436.

[25] Mohammad Rakibul Islam , Md. Abu Jamil, Md. Siraz-Uz Zaman, Syed Asir Hamim Ahsan, Mahfuz Kabir Pulak, Fariha Mehjabin, Md Moinul Islam Khan, Jubair Alam Chowdhury, Mohibul Islam," Design and analysis of birefringent SPR based PCF biosensor with ultra-high sensitivity and low loss", Optik, Volume 221, November 2020

[26] Md. Biplob Hossain, S.M. Riazul Islam, K.M. Tasrif Hossainc, Lway Faisal Abdulrazak, Md. Nazmus Sakib, I.S. Amiri, " High sensitivity hollow core circular shaped PCF surface plasmonic biosensor employing silver coat: A numerical design and analysis with external sensing approach" Results in Physics, Volume 16, March 2020

[27] Md. Biplob Hossain, T. V. Mahendiran, Lway Faisal Abdulrazak, Ibrahim Mustafa Mehedi, Md. Amzad Hossain, Md. Masud Rana, " Numerical analysis of gold coating based quasi D-shape dual core PCF SPR sensor" Optical and Quantum Electronics, 30 September 2020

[28] Amit KumarShakya, SurinderSingh, " Design of dual polarized tetra core PCF based plasmonic RI sensor for visible-IR spectrum", Optics Communications, Volume 478, 1 January 2021

[29] Yaser Esfahani Monfared, Montasir Qasymeh, " Plasmonic Biosensor for Low-Index Liquid Analyte Detection Using Graphene-Assisted Photonic Crystal Fiber" Plasmonic, 11 January 2021 
Figures
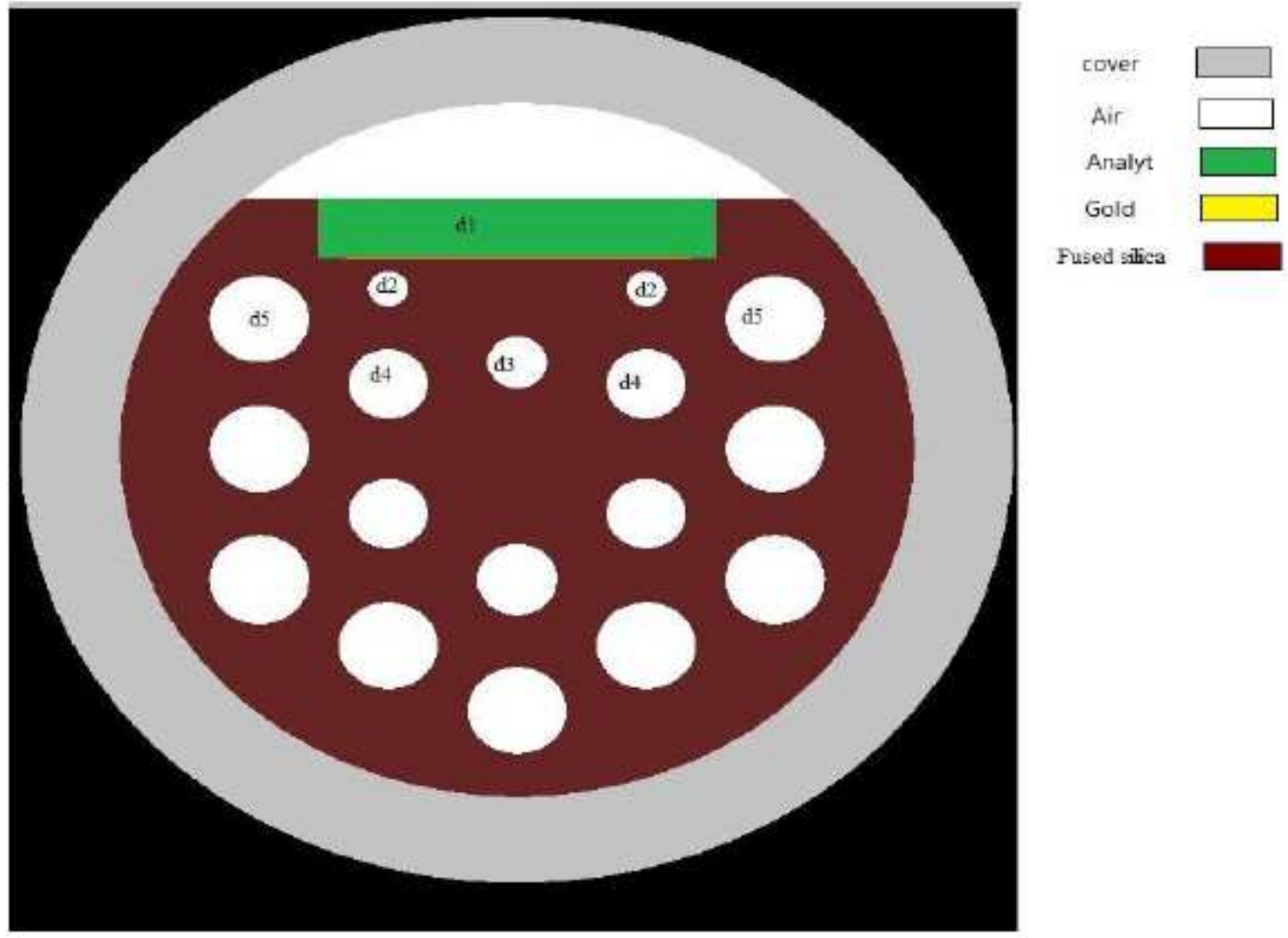

Fused silica

Figure 1

Cross-section of the proposed PCF-based SPR biosensors 


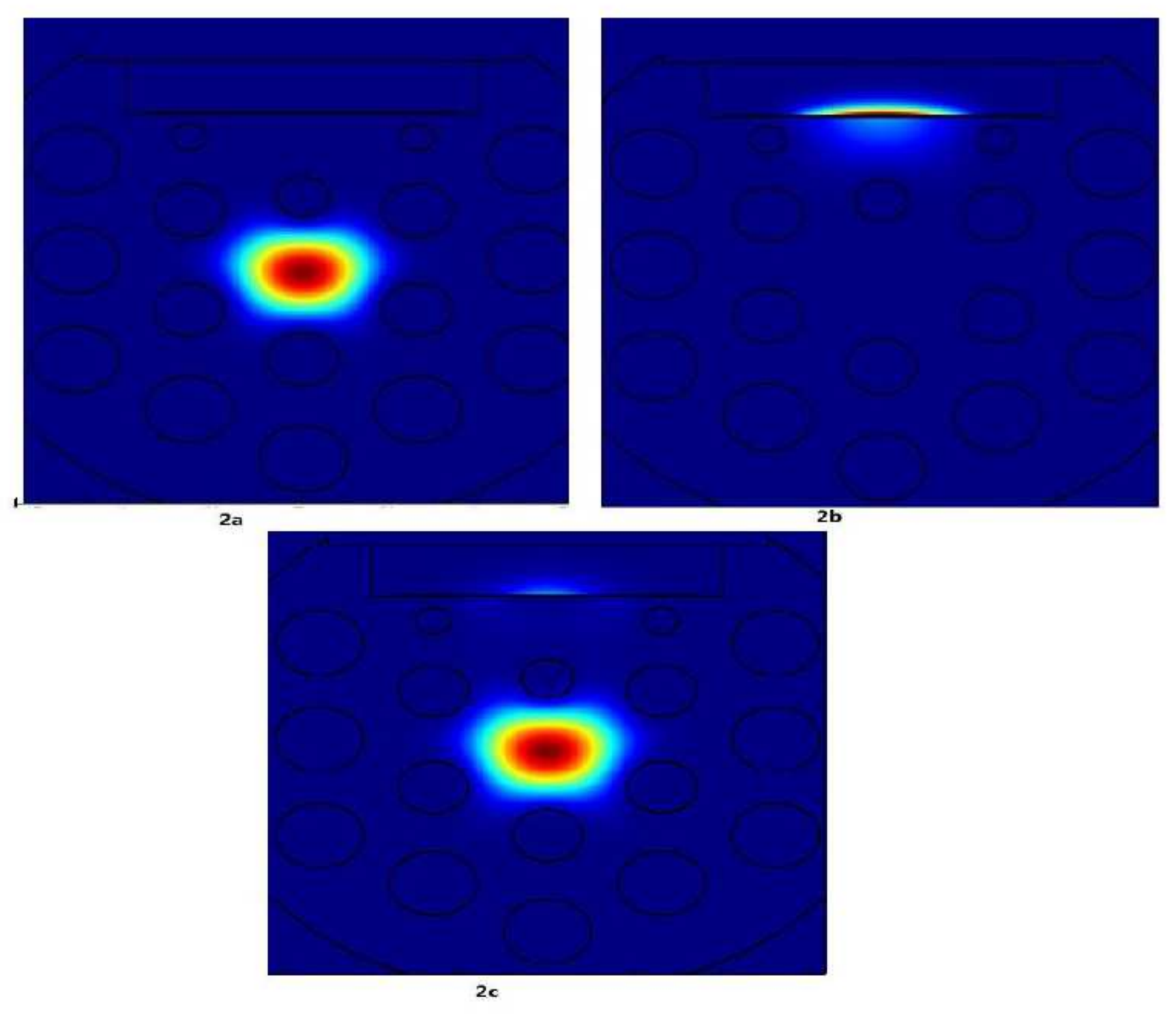

\section{Figure 2}

Light energy flow distributions for the analyte with $n=1.4,2 a$ ) are the field distributions in FM mode, 2b) SPP mode, 2c) phase matching modes 


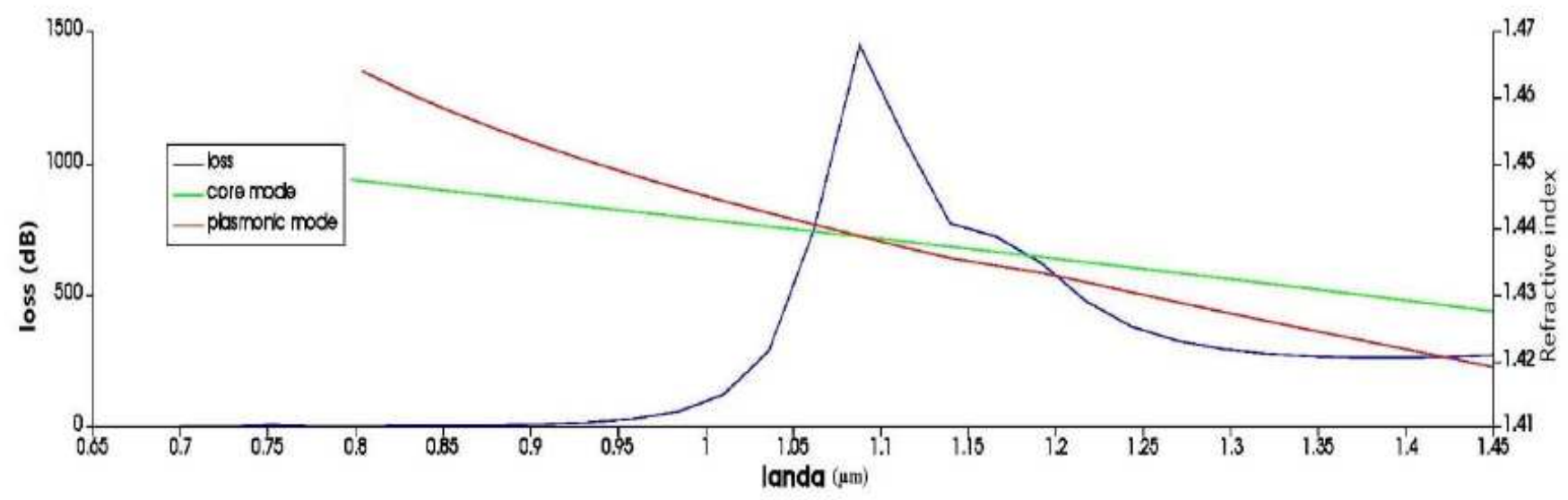

Figure 3

The confinement loss, dispersion relations of FM and plasmonic mode for na=1.4

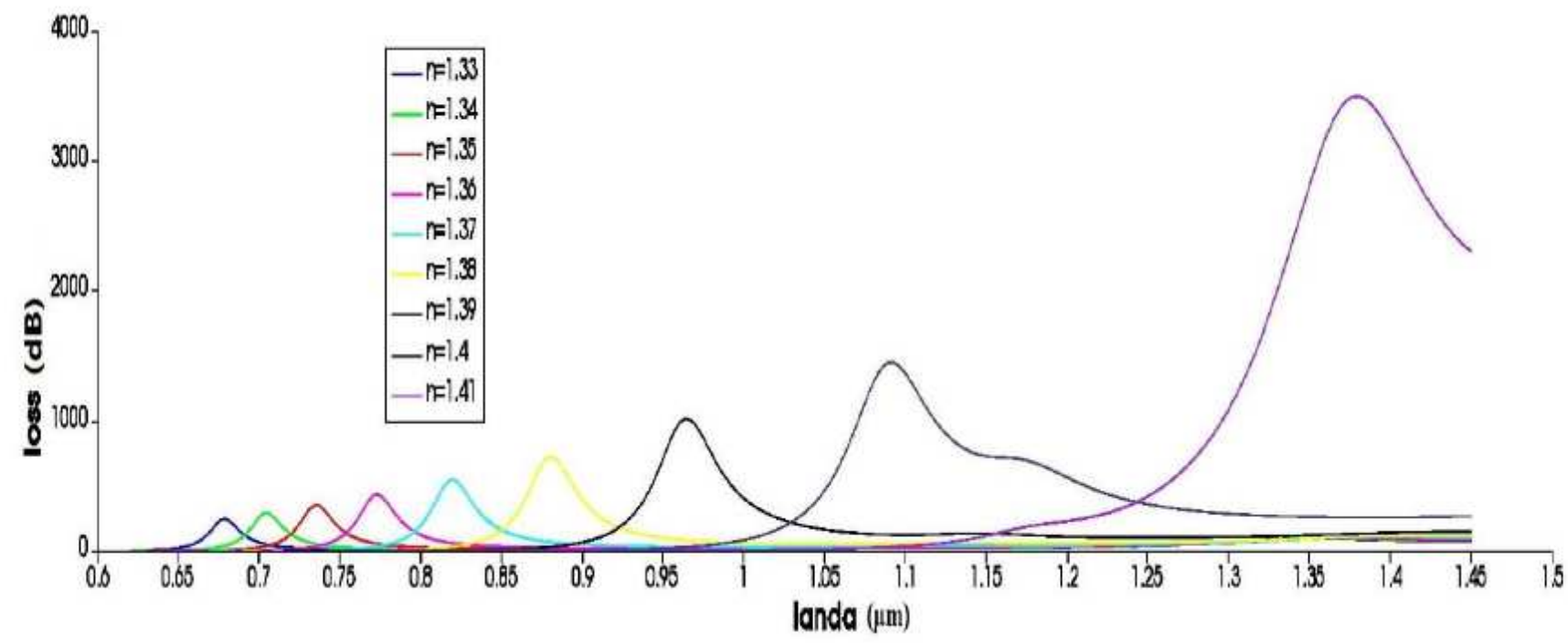

Figure 4

Loss spectra with increasing analyte RI from 1.33 to 1.41 


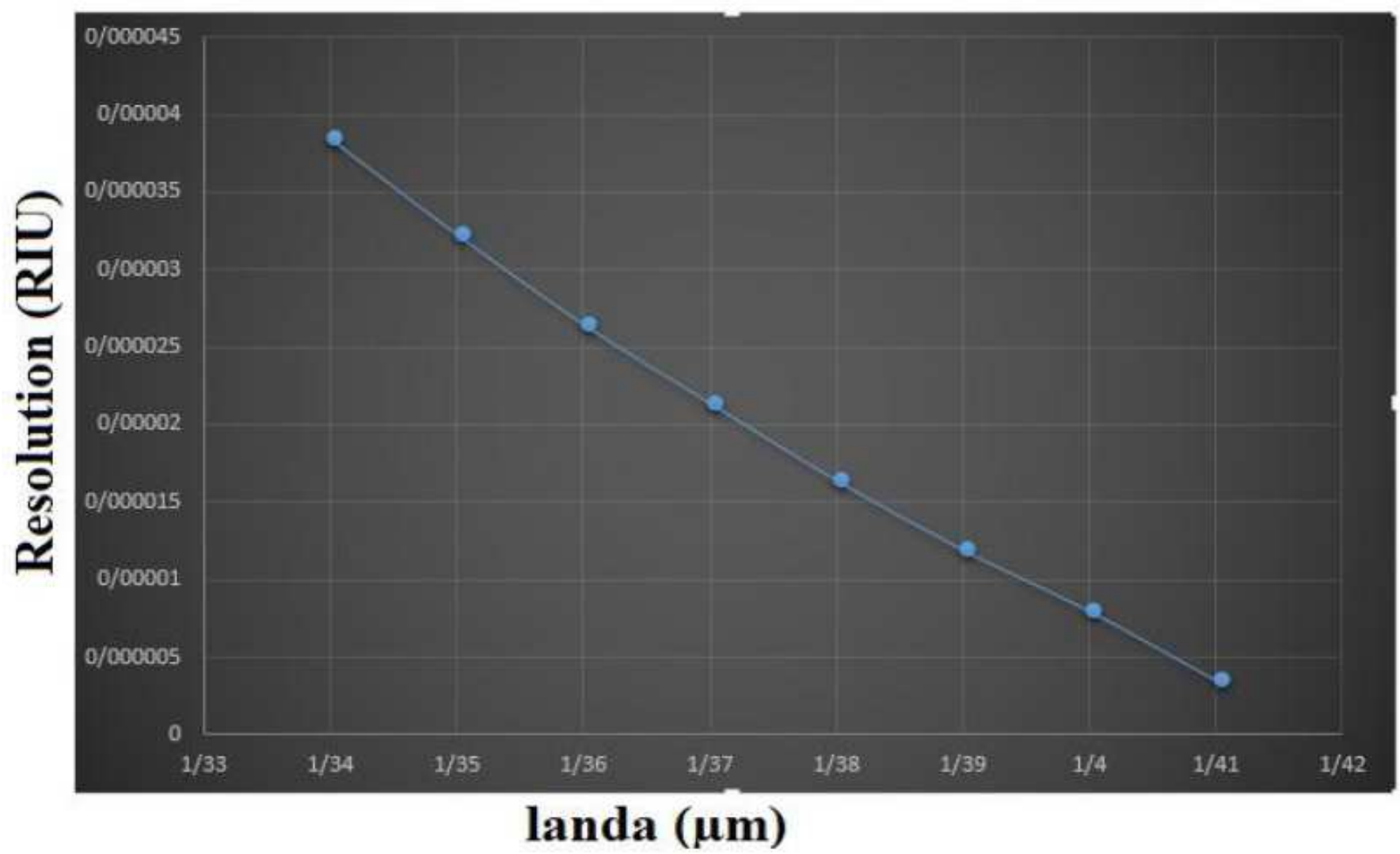

Figure 5

Resolution versus for different analyte's RI 


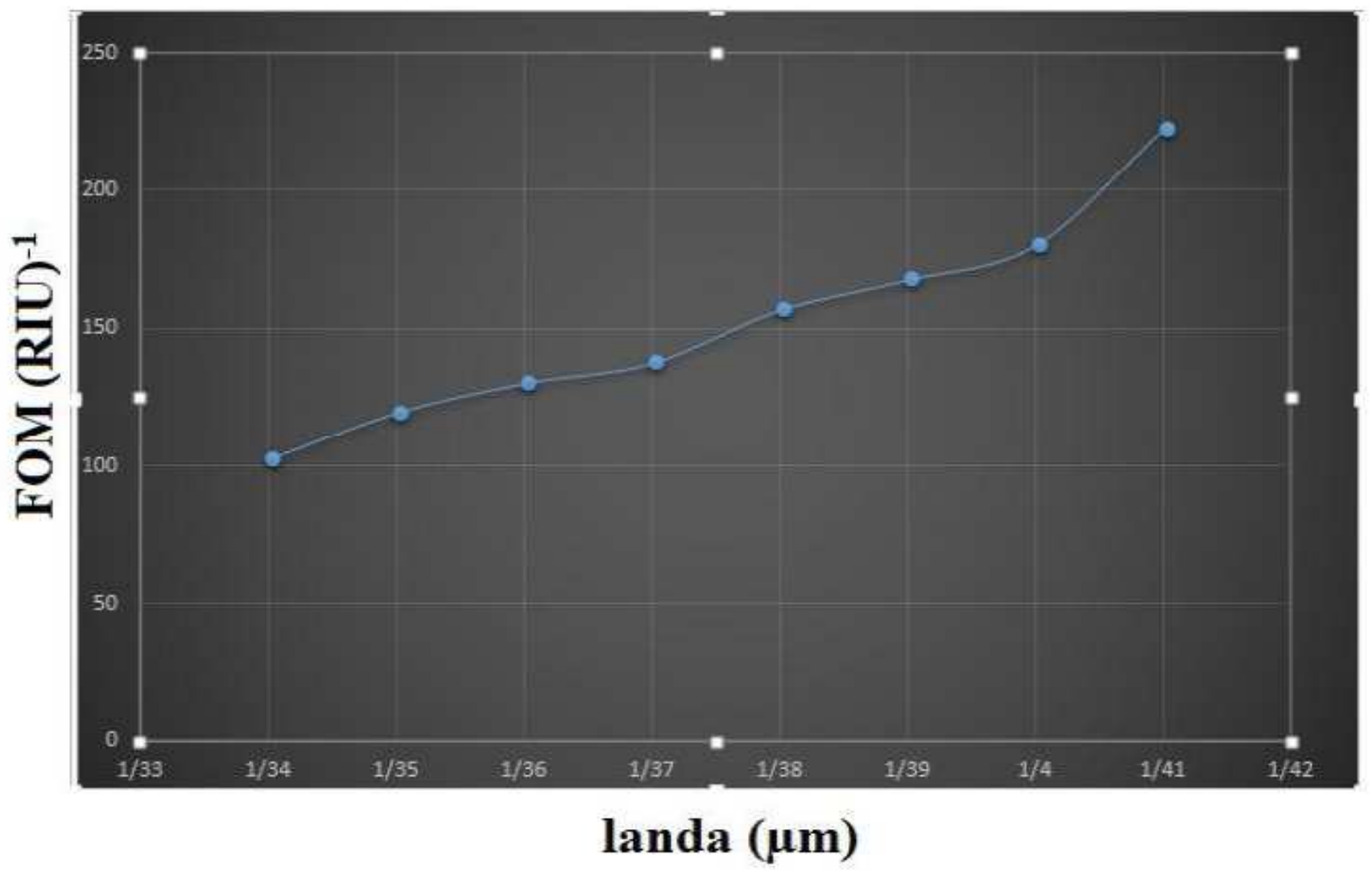

Figure 6

FOM versus for different analyte's RI

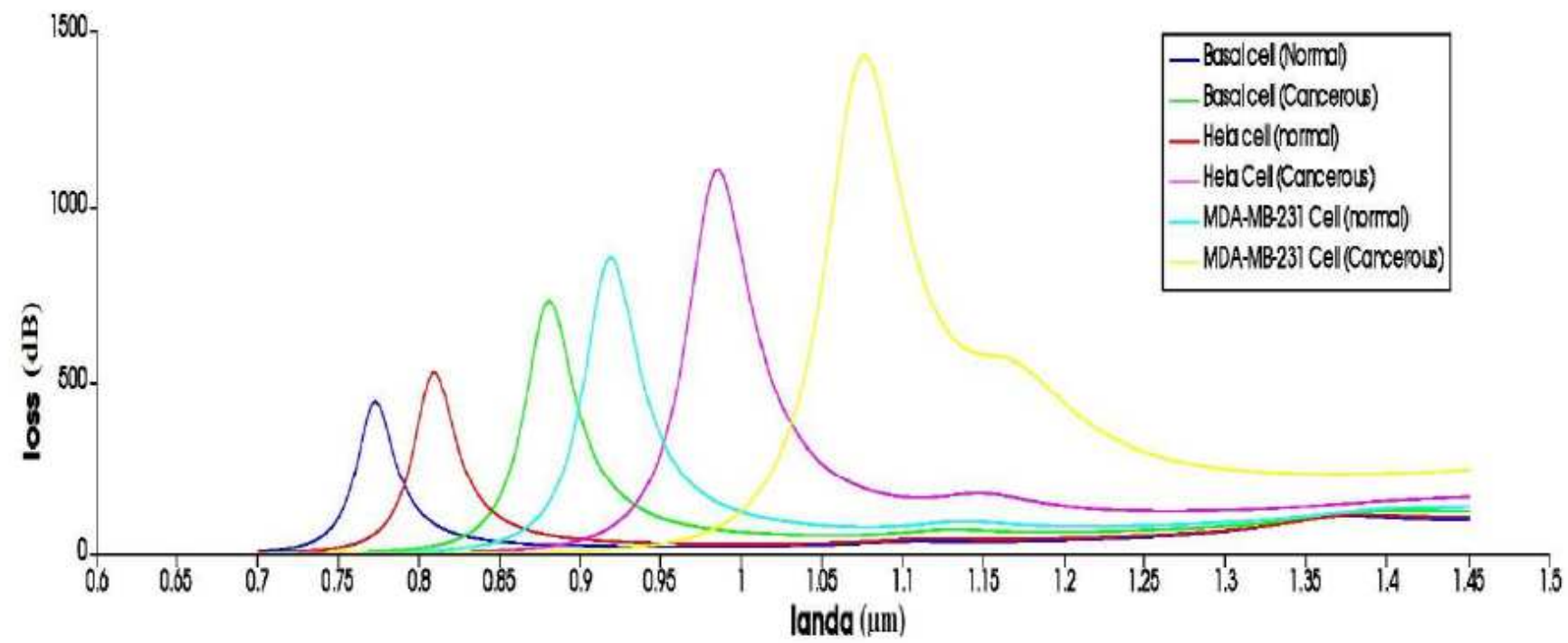

Figure 7

Loss spectra for Normal and cancer cell 